\title{
CONTRIBUTION A LA CONNAISSANCE ÉPIDEMIOLOGIQUE ET MYCOLOGIQUE DES TEIGNES INFANTILES DE TUNISIE
}

\section{Enquête menée principalement chez des écoliers musulmans}

\author{
Par F. Coutelen, G. Cochet, J. Biguet, S. Mullet, \\ M. DOBY-DUBOIS et S. DEBLOCK
}

A partir de 1950, l'Institut des Hautes-Etudes Tunisiennes nous avait chargés d'une enquête sur les teignes des enfants. Deux missions se succédèrent en septembre-octobre 1950 et 1953 , qui parcoururent la plus grande partie de la Régence, à la seule exception importante de Gabès et de l'île de Djerba.

Dans les pages qui suivent, nous exposerons nos constatations épidémiologiques et l'étude mycologique des prélèvements effectués, en les comparant aux résultats des travaux similaires entrepris par divers auteurs dans les autres pays du Nord de l'Afrique.

\section{A. ETUDE ÉPIDÉMIOLOGIQUE}

La mission de 1950 a prospecté essentiellement les grandes villes còtières et Kairouan ; celle de 1953, de petites agglomérations et des villages de l'intérieur.

Dans ces conditions, il nous semble préférable de présenter séparément les résultats obtenus, et ceci d'autant plus que l'organisation de ces missions fut un peu différente, la seconde ayant profité de l'expérience de la première. En effet, lors de notre premier séjour en Tunisie, nous avons désiré ramasser le plus grand nombre possible de prélèvements. Ceci nous a amenés à faire porter notre enquête sur plus de 16.000 écoliers. Mais, compte tenu des difficultés de mise en route et du temps limité dont nous disposions, le tiers seulement de ces enfants fut examiné par nos soins; les autres, pour aller plus vite, furent au préalable triés par un personnel toujours très dévoué, mais qui n'était pas nécessairement spécialisé. Il est fort possible et même probable que, dans ces conditions, des lésions discrètes ou masquées par l'impétigo soient passées inaperçues; ce fait a dû retentir sur le taux d'infestation global observé.

ANN. de Parasitologie, T. XXXI, No $4 .-1956$. 
Par contre, nous avons limité l'ampleur de l'enquête de notre deuxième voyage, mais les 8.000 enfants qu'elle a approximativement intéressés sont tous passés entre nos mains. Il s'ensuit que le taux d'infestation auquel nous arrivons doit correspondre sensiblement à la réalité et on verra qu'il est beaucoup plus élevé que celui de 1950.

Enfin, nous avons eu essentiellement affaire à des écoliers musulmans (23.746) et rarement à des enfants israélites, français ou italiens (5 à 600 seulement).

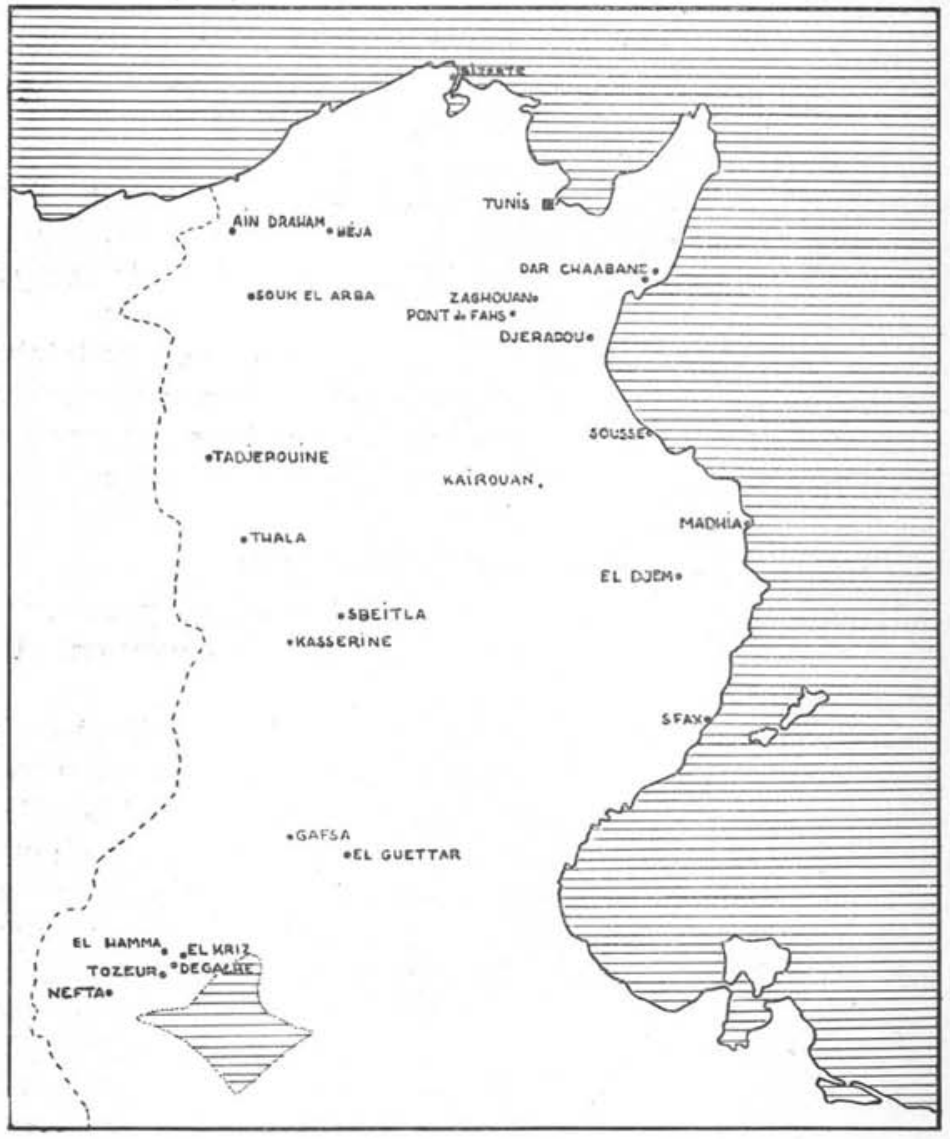

Carte de Tunisie

Situation des villes, bourgs et villages prospectés au cours de l'enquàte sur les teignes infantiles au cours des deux missions. 
Nous avons été ainsi incités à exposer nos observations épidémiologiques et cliniques en trois tableaux : le premier intéressant les écoliers musulmans prospectés en 1950 ; le second, les écoliers musulmans examinés en 1953 , et, enfin, le troisième qui rassemble les résultats très fragmentaires fournis par les deux missions concernant les enfants non musulmans. Cette façon de procéder a l'avantage de grouper les musulmans qui, dans l'ensemble, ont un genre de vie très comparable, ce dernier facteur influençant, selon toute vraisemblance, beaucoup plus la fréquence et la nature des teignes d'une région donnée que la race des individus (il ne faut d'ailleurs pas oublier que la dénomination « musulman » est seulement religieuse et s'applique à des groupements ethniques qui peuvent être fort divers).

\section{I. - La teigne chez les écoliers musulmans}

\section{$1^{\circ}$ Résultats de la mission de 1950: Commentaire du tableau I}

Cette mission a intéressé les grandes villes côtières : Tunis et sa banlieue, Bizerte, Sousse, Madhia, Sfax, et une ville de l'intérieur : Kairouan, ainsi qu'une agglomération de moindre importance : Pont-du-Fahs.

Sur 16.381 enfants des écoles, 1.157 , soit $7,06 \%$, furent trouvés parasités. Les formes cliniques rencontrées sont des Trichophyties chez 70,18\% des teigneux, des Favus chez 24,89\%, des associations de Trichophyties et de Favus dans 4,40\% des cas. La Microsporie est rarissime (une seule observée). Nous n'avons jamais vu de Kérions, bien que, six fois, des Ctenomyces aient été isolés par culture. Il s'agissait peut-être de lésions trop peu évoluées pour être caractéristiques ou plus vraisemblablement de lésions assez discrètes et cachées par une impétiginisation souvent intense.

Si l'on compare maintenant la répartition des formes cliniques dans la population scolaire des villes de la côte (14.284 enfants examinés) et des villes de l'intérieur qui se ramènent en fait à Kairouan (1.957 enfants sur 2.097 examens), on constate la prédominance des Trichophyties dans le premier groupe $(75,67 \%$ contre $19,69 \%$ faviques) et celle du Favus dans le second groupe $(56,79 \%$ contre $36,41 \%$ trichophytiques seulement).

Ce phénomène s'explique vraisemblablement par l'organisation sanitaire plus poussée des villes côtières, qui disposent d'un personnel qualifié relativement nombreux et d'un équipement assez important. 
La situation particulière des principales villes prospectées mérite quelques commentaires :

A Tunis et dans sa banlieue, nous avons personnellement passé en revue 5.833 écoliers (non compris 14 enfants vus dans les dispensaires), dont 2.120 fréquentaient des écoles franco-musulmanes de banlieue (La Marsa et L'Ariana), 3.040 des écoles coraniques et 673 des kouttabs. Sur cet ensemble, 224 seulement étaient parasités, soit $3,84 \%$. Cependant, dans cette ville privilégiée, la répartition clinique reste sensiblement celle de l'ensemble de la population scolaire prospectée par cette première mission : 86,16\% des cas sont des Trichophyties, 11,60\% des cas sont des Favus.

Le taux de l'infestation est beaucoup plus élevé dans les kouttabs où l'hygiène nous a dans l'ensemble paru moindre que dans les autres écoles; sur 673 enfants, $12,18 \%$ étaient porteurs de lésions trichophytiques et $9,75 \%$ de lésions faviques.

La seule teigne microsporique diagnostiquée au cours de nos deux missions chez un enfant musulman le fut en 1950 à Tunis.

Au total, la situation de l'endémie teigneuse est très favorable à Tunis, où se rencontrent évidemment des moyens médicaux considérablement supérieurs à ceux du reste du pays.

Une fois quittée la capitale, on constatera que le pourcentage des enfants parasités va s'élever en dépit des modalités moins favorables du dépistage, la sélection des suspects n'ayant pas été assurée habituellement par nous : il est de 14,19\% à Bizerte, de 10,14\% à Sousse, de 11,50\% à Madhia et de 5,40\% seulement à Sfax.

A Bizerte et à Sousse, la répartition des variétés cliniques est pratiquement celle de Tunis. Il faut d'ailleurs noter qu'à Sousse, un examen particulièrement minutieux de deux écoles, groupant une population de 960 filles, a révélé l'existence d'une endémie de teigne trichophytique sévère, puisque, sur 163 enfants parasités (soit 16,96\%), 157 portaient des lésions trichophytiques souvent extrêmement discrètes, cinq du Favus, et une les deux types de lésions associées.

A Sfax, le pourcentage total des enfants dépistés était voisin de celui de la capitale $(5,40 \%)$. La discrétion de l'endémie s'explique, en partie du moins, par l'intérêt porté par M. le Docteur Jadfar, Médecin-chef du Dispensaire polyvalent d'Hygiène scolaire, à la thérapeutique et à la prophylaxie de la teigne. Les enfants trichophytiques sont systématiquement traités par l'acétate de thallium ; les cheveux des enfants sont régulièrement tondus à l'école même, tandis que les instituteurs luttent contre le port de la chéchia, facteur de propagation des teignes. Une des conséquences du traite- 
ment systématique des trichophytiques est le relèvement du taux des infestations faviques qui représentent $23,52 \%$ du total des enfants dépistés.

Pour des raisons toutes différentes, croyons-nous, cette évolution dans la distribution relative des teignes va s'accentuer à Madhia où la fréquence du Favus $(43,74 \%)$ atteint presque celle des Trichophyties $(46,59 \%)$. La pauvreté de la population de cette ville nous a semblé, en effet, très grande et, par ailleurs, très insuffisants les moyens médicaux.

La même situation économique, sociale et sanitaire entraìne peut-être un aspect épidémiologique comparable à Kairouan où le pourcentage des Favus $(56,20 \%)$ dépasse cette fois celui des Trichophyties $(36,53 \%)$.

\section{$2^{\circ}$ Résultats de la mission de 1953 : Commentaire du tableau II}

Cette deuxième mission a parcouru 18 villes et villages de l'intérieur, à savoir : Béja, Aïn-Draham, Souk-el-Arba, Tadjerouine, Thala, Sbeitla, Kasserine, Gafsa, El-Guettar, Tozeur, Degache, ElKriz, Nefta, El-Hamma, El-Djem, Dar-Chaabane, Djéradou, et Zagouan.

7.330 écoliers furent examinés soigneusement par nous (à l'exception de 350 à Souk-el-Arba). Parmi eux, 17,09\% étaient teigneux. Nous expliquons en partie ce pourcentage global nettement supérieur à celui obtenu lors du premier voyage $(7,43 \%)$ par les modalités de dépistage, mais aussi par le fait que la première mission avait traversé les villes les mieux équipées du pays au point de vue sanitaire.

Les teignes se répartissent ainsi :

- Trichophyties ........... 13,06 \% des cas examinés.

- Favus .................. $3,56 \%$

- Favus et Trichophyties ....... $0,47 \%$

Et si l'on considère les pourcentages des variétés cliniques en fonction de l'ensemble des enfants parasités, on trouve que 76,38\% des teigneux étaient trichophytiques, 20,83\% faviques et 1,91\% porteurs des deux teignes associées. Ces chiffres correspondent sensiblement à ceux trouvés pour l'ensemble des villes côtières (respectivement $75,67 \%, 19,69 \%, 4,02 \%$ ) ; on ne retrouve pas l'inversion du rapport Favus-Trichophytie si nette à Kairouan. 
Mais il est vrai que cette seconde mission n'a parcouru que de petites villes et villages ( 7.330 écoliers pour 18 agglomérations en 1953 contre 16.400 pour 7 seulement en 1950), où la promiscuité est peut-être moindre que dans les quartiers pauvres des grosses agglomérations.

Nous avons observé le taux d'endémie le plus élevé à Djéradou, petit village isolé sur un plateau montagneux, avec $42 \%$ des écoliers parasités. Le pourcentage des teigneux s'échelonne, dans les autres agglomérations, de 7,40 (Souk-el-Arba) à 29,07\% (El-Djem).

Dans chaque ville ou village, on retrouve généralement une répartition des formes cliniques à l'image de la répartition globale. Les exceptions, telles qu'à El-Kriz et El-Hamma, où le taux des faviques dépasse $60 \%$, sont peu significatives, compte tenu du petit nombre d'enfants examinés (qui représentait, du reste, à peu de chose près, la population infantile de ces villages); dans ces localités, la population ne semblait d'autre part pas plus misérable qu'ailleurs. De fait, les populations scolaires visitées au cours de ce second voyage nous ont donné l'impression d'un ensemble homogène quant aux conditions sociales et sanitaires.

Nous retiendrons cependant, sans pouvoir l'expliquer, la rareté du Favus à Béja, où il ne représente que 3,48\% des teignes.

\section{Influence du sexe et de l'âge des enfants}

En 1953, 5.701 garçons furent examinés contre 1.629 filles : $17,98 \%$ des garçons étaient parasités et $13,99 \%$ des filles ; le taux d'infestation était done très comparable.

La Trichophytie, qui prédomine dans les deux sexes, est un peu plus fréquente chez les filles que chez les garçons $(91,22 \%$ des filles teigneuses contre $73,07 \%$ des garçons teigneux), tandis que le Favus semble atteindre les garęons avec prédilection $(24,48 \%$ des garçons parasités pour 4,82\% des filles seulement). Nous n'avons pas voulu établir cette statistique en tenant compte des populations scolaires de la première mission, car nous avions omis pour certaines écoles de nous renseigner sur l'effectif exact des filles et des garȩons. Cependant, les chiffres dont nous disposons pour 1950 confirment les résultats précédents.

Nous avons connu avec quelque certitude, lors de la mission de 1953, l'àge de 4.322 enfants et adolescents de 6 à 17 ans. Le pourcentage total des écoliers parasités, qui est de $20,67 \%$ pour les enfants de 6 ans, reste sensiblement du mème ordre de grandeur jusqu'à l'âge de 12 ans, à partir duquel il commence à décroître 
(13 \% environ à 13-14 ans), pour tomber brusquement à 15 et 16 ans (5 et $8 \%$ ). Cette chute du taux d'infestation est essentiellement le fait de la trichophytie qui atteint 15 à $16 \%$ des enfants âgés de 6 à 9 ans et régresse assez progressivement par la suite (8,36 \% à 13 ans, 2,5 \% à 15 ans). Quant au Favus, il s'observe chez environ 3 à $6 \%$ des enfants et des adolescents examinés et semble assez indifférent à l'âge, du moins dans les limites de notre travail.

\section{II. - La teigne chez les écoliers non musulmans}

\section{Missions 1950 et 1953 réunies : Gommentaire du tableau III}

En 1950, un certain nombre de jeunes Français et Israélites furent examinés soit à l'école, soit le plus souvent dans les consultations ; parmi eux, 10 Européens et 22 Israélites étaient parasités; en 1953, sur 287 écoliers européens, aucun ne fut trouvé infesté ; sur 121 israélites, 8 étaient teigneux.

Le nombre d'enfants intéressés par notre statistique est trop peu important et manque par trop d'homogénéité pour prêter à de nombreux commentaires.

Le faible pourcentage d'infestation des Européens ne surprend pas, leur niveau social étant habituellement très supérieur à celui des autres enfants. Parmi 40 teignes dépistées chez les écoliers israélites et européens, on ne compte que deux Favus (enfants italiens). Par contre, elles comprennent deux Microspories (un Français et un Italien), sur trois découvertes dans l'ensemble de nos deux prospections.

En ce qui concerne la teigne chez les enfants israélites, M. le Professeur Béretvas a eu l'extrême amabilité de nous communiquer à l'époque (1950) une statistique effectuée par les Services de l'O.S.E. dans 16 villes et oasis du Sud-Tunisien (Gabès, Harakebira, Harasrira, Tatahouine, Zarzis, Ben-Gardane, Médénine, Gafsa, Kébili, El-Hamma, Menzel, Douz, Matmata, Nefta, Tozeur, Tamezret) ; elle portait sur 2.967 enfants, correspondant à une population de 11.376 habitants; 321 , soit $10,81 \%$, étaient teigneux ; 32 seulement, soit 9,96\% des enfants parasités, étaient porteurs de lésions faviques; les autres étaient atteints de Trichophyties (à quelques Microspories près, peut-être, les deux formes de tondantes n'étant pas distinguées dans la statistique). Le Favus est donc moins fréquent en Tunisie chez les enfants israélites que chez les enfants musulmans. 


\section{Etude comparée de l'endémie teigneuse}

\section{dans le pays d'Afrique du Nord}

Les principales enquêtes effectuées à notre connaissance au Maroc espagnol et français, en Algérie, en Tunisie, en Tripolitaine et en Egypte, ont été résumées dans le tableau IV.

Il est difficile de comparer l'endémie globale dans ces divers pays ; outre qu'elle n'est pas toujours signalée par les auteurs, elle dépend de la modalité de la prospection, et l'interprétation comparée des statistiques doit être prudente. Il est cependant évident que l'endémie est partout importante et peut varier beaucoup suivant le groupement humain que l'on considère ; c'est ainsi qu'au Maroc, elle est très différente chez les Musulmans, les Juifs ou les Européens. Le Favus est deux fois moins fréquent chez les jeunes Israélites que chez les jeunes Musulmans. Mais en fait, selon toute vraisemblance, la condition sociale des individus importe seule avec les moyens médicaux thérapeutiques et prophylactiques mis à leur disposition, et la race n'intervient que dans la mesure où elle est liée à un standing de vie. C'est ainsi que les Israélites, parasités seulement dans 9,9\% des cas au Maroc (statistique 1948-1949), le sont dans 33,5 \% des cas en Tripolitaine. Quant aux Européens, leur situation souvent aisée explique parfaitement leur faible pourcentage d'infestation.

Nous n'avons presque aucun renseignement concernant l'évolution de l'endémie dans le temps ; les statistiques du Service central de l'Hygiène scolaire rapportées par Sicault, Gaud et Faure montrent toutefois une diminution du nombre des teigneux au Maroc entre les années 1938-1939 et les années 1948-1949.

Favus et Trichophyties, parfois associés (les associations doivent être en fait beaucoup plus fréquentes qu'on ne le signale), représentent la presque totalité des teignes nord-africaines, quelle que soit la région considérée. Les Microspories sont toujours très rares et les Kérions exceptionnels.

La répartition des formes cliniques montre que la fréquence relative du Favus diminue progressivement du Maroc à la Tunisie (de $76,6 \%$ à $22,71 \%$ ); il disparait presque en Tripolitaine $(1,7 \%)$, mais, en Egypte, il représente de nouveau un pourcentage élevé des cas de teigne (32\%). Peut-être la faible densité de la population tripolitaine, moins favorable à la promiscuité nécessaire à la contagion du Favus, explique-t-elle en partie sa rareté.

Au total, la teigne en Afrique du Nord est caractérisée par sa fréquence et le peu de variété de ses formes cliniques habituelles. 


\section{B. ETUDE MYCOLOGIQUE}

Avant d'exposer nos résultats, il est nécessaire de préciser brièvement la position que nous avons adoptée concernant la systématique des Dermatophytes.

La variabilité indéniable des aspects macroscopiques (et parfois microscopiques) des champignons de ce groupe (1) nous a incités à adopter dans l'ensemble les synonymies d'especes acceptćes par Conant et ses collaborateurs (1954), qui complètent et modifient celles qui furent proposées en 1934 par Emmons ; elles apportent, nous semble-t-il, de la clarté dans un chapitre de la mycologic médicale particulièrement confus, qui s’alourdissait encore chaque jour d'espèces nouvelles souvent discutables; l'émiettement des espèces avait encore comme conséquence regreltable d'inciter les mycologues isolant des souches dont certains caractères se révélaient communs à plusieurs espèces voisines, à prendre l'une des deux décisions suivantes: ou bien ils rattachaient leur souche à l'une ou à l'autre de ces espèces, après de longues hésitations peut-être, mais sans être toujours absolument certains du bienfondé de leur diagnose; ou bien ils créaient une espèce nouvelle supplémentaire à l'occasion de cette souche légèrement aberrante. On pourrait reprocher aux multiples synonymies créées par les auteurs américains d'être un peu simplistes, d'entraìner une excessive imprécision et d'encourager à une étude superficielle des cham-

(1) Josserand, en 1951, écrivait: \& On a l'impression que les champignons supérieurs (les autres aussi peut-être mais je ne les connais point) n'ont pas atteint la stabilité relative des autres êtres : il semble qu'ils ne soient pas fixés, qu'ils soient encore dans la phase évolutive, qu'ils aient gardé une certaine aptitude à muter, à engendrer des formes nouvelles. \$

Les apparences sont, nous semble-t-il, également en faveur de cette hypothèse en ce qui concerne les Dermatophytes (Vanbreuseghem et Van Brussel n'ont-ils pas obtenu en 1950 un mutant de Langeronia soudanensis en le cultivant sur milieu à la terre ?).

Or, à ces mutations qui modifient des caractères morphologiques déjà peu nombreux dans un groupe aussi homogène et eréent ainsi des souches légèrement différentes de celles dont elles sont issues, viennent encore s'ajouter, pour compliquer les diagnoses et favoriser la création d'espèces nouvelles, des variations d'aspects culturaux parfois considérables pour une même souche : ces variations dépendent du moment de l'ensemencement, du tube de culture considéré et de l'origine des produits utilisés pour faire les milieux ; elles peuvent se manifester avec une particulière évidence au cours des subcultures.

On peut lire d'ailleurs dans la conclusion d'un article de Catanei, paru en 1953 et traitant du champignon du favus, les deux phrases suivantes dont nous inversons l'ordre : \& Pour beaucoup de champignons parasites étudiés au laboratoire, à la notion de fixité des caractères culturaux, il faut substituer, en effet, celle d'instabilité relative qui les amène à la dégradation morphologique, ou au contraire à des changements qui accentuent la valeur de certains caractères morphologiques. » Avant de faire d'un type cultural non encore observé une espèce nouvelle, il faudra prendre toute garantie qu'il ne s'agit pas d'une culture modifié ou en voie de modifieation. » 
pignons isolés. C'est pourquoi nous préférons conserver comme des variétés, à l'intérieur de l'espèce collective, toutes les espèces tombées en synonymie, à condition toutefois que la stabilité et la fixité de quelques caractères distinctifs nous semblent suffisamment affirmées pour justifier leur individualisation. Nos diagnoses s'effectuent par conséquent en deux temps : d'abord identification de la souche étudiée à l'une des grandes espèces acceptées par Conant et ses collaborateurs, et, secondairement, mais seulement dans les cas favorables, rattachement de la souche à une variáté plus précise.

Par contre, en matière de dénominations génériques, nous n'avons pas eu l'impression que les vues unicistes aient constitué un réel facteur de progrès. En particulier, le vaste groupe des Trichophyton, tel qu'il a été compris par Emmons (1934), est manifestement hétérogène, ainsi que le souligne très justement Vanbreuseghem (in Langeron et Vanbreuseghem, 1952). Dès lors, il est peut-être inutile, pour l'instant du moins, d'y rattacher le vieux genre Achorion Lebert, 1843, - bien que, Lotaniquement et dans l'état actuel de nos connaissances, cette assimilation soit parfaitement défendable - car ce nom évoque immédiatement à l'esprit une seule espèce déterminant, à de rarissimes exceptions près, tous les Favus humains.

Nous avons moins d'hésitation en ce qui concerne les Trichophyton microïdes qui constituent un groupe assez nettement individualisé ; aussi les classons-nous dans le genre Ctenomyces Eidam. 1888, suivant l'exemple de Vanbreuseghem, tout en reconnaissant d'ailleurs avec cet auteur (in Langeron et Vanbreuseghem, 1952) que le rattachement des microïdes au genre Ctenomyces, proposé par Langeron et Milochevitch en 1930, n'est pas parfaitement justifié.

Enfin, nous acceptons, pour l'immédiat et sous réserve de recherches ultérieures, la validité du genre Langeronia créé par Vanbreuseghem en 1950 .

Sur 2.483 prélèvements positifs, correspondant à 2.443 enfants musulmans et 40 seulement non-musulmans, 1.591 ont donné lieu à des cultures positives ( 1.574 pour les Musulmans et 17 pour les autres).

Les espèces isolées sont les suivantes (tableau V) :

Achorion schönleini (Lebert, 1843).

Trichophyton violaceum Bodin, 1902.

T. tonsurans Malmsten, 1845. 
Langeronia soudanensis (Joyeux, 1912).

Ctenomyces mentagrophytes (Robin, 1853).

Microsporum canis Bodin, 1902.

\section{$1^{\circ}$ Genre Trichophyton Malmsten, 1845.}

1.067 souches se rapportaient à ce genre, dont 1.050 sont des $T$. violaceum, qui représentent par conséquent, avec le pourcentage de $98,40 \%$, la presque totalité des Trichophyton isolés.

Sur ces 1.050 souches, 98 avaient été d'abord rapportées à la variété glabrum décrite en 1900 par Sabouraud ; l'examen très prolongé des cultures et des subcultures de nos souches ne nous a pas paru justifier l'individualité de cette dernière variété ; nous exposerons dans une note séparée nos observations à ce sujet.

Les 17 dernières souches se rattachaient au groupe des Trichophyton cratériformes et nous les avons étiquetées T. tonsurans, compris suivant Conant et ses collaborateurs (1954), qui font tomber en synonymie devant $T$. tonsurans les espèces $T$. sabouraudi Blanchard, 1896, T. epilans Boucher et Mégnin, 1887, et T. sulfureum Sabouraud, 1910. Trois souches avaient pris d'emblée l'aspect de colonies en cratères qui imposaient le diagnostic de $T$. tonsurans; une quatrième souche était déjà un peu atypique, car très plate ; une cinquième souche ne devint cratériforme qu'après passage sur le milieu de conservation; une sixième souche, nettement acuminée et que l'on aurait été tenté d'appeler $T$. sabouraudi, a donnć deux ans plus tard des colonies franchement en cratère ; quant aux autres souches, elles étaient plus atypiques encore : leur aspect a varié avec les repiquages et elles n'ont été acuminées ou cratériformes que très épisodiquement et sur quelques colonies seulement.

Les cultures variaient du blane au bistre, mais l'une d'entre elles fut toujours beaucoup plus jaune, cette pigmentation s'avérant particulièrement intense dans des subcultures réalisées à partir de cultures sur milieu à la terre de Vanbreuseghem et Van Brussel.

Par conséquent, les faits observés confirment la variabilité morphologique des cultures des champignons de ce groupe et apportent des arguments à la théorie uniciste des auteurs américains.

$2^{\circ}$ Genre Achorion Lebert, 1845.

Nous avons étudié 546 souches d'Achorion schönleini, dont 544 provenaient d'enfants ou d'adolescents musulmans. Si nous avons constaté l'extrême polymorphisme de ce champignon, nous n'avons pas, par contre, retrouvé les jordanons décrits par Langeron et Baéza en 1930. La même souche peut, en effet, présenter 
dans le même tube de cultures plusieurs des aspects décrits par ces auteurs comme des formes fixes. L'existence des jordanons a d'ailleurs été niée par Catanei dans un travail très documenté paru en 1953.

$3^{\circ}$ Genre Langeronia Vanbreuseghem, 1950.

Une souche unique de $L$. soudanensis fut isolée à Bizerte. Il s'agit très vraisemblablement d'un élément importé d'autres régions d'Afrique où cette espèce est assez fréquente (Soudan anglo-égyptien, Congo, Cameroun, etc...).

Dans certaines de nos cultures sur lames âgées de trois semaines, les aleuries vraies étaient parfois très nombreuses, contrairement à ce que dit Vanbreuseghem (in Langeron et Vanbreuseghem, 1952), qui ajoute dans la diagnose du genre que les arthrospores peuvent simuler des grappes, mais que les aleuries disposées suivant le type Acladium sont rares.

Précisons que nos observations ont été réalisées non seulement sur des préparations fixées et colorées, mais encore à frais et sans lamelles, pour étudier les hyphes dressées et éviter la confusion entre aleuries et arthrospores.

Joyeux d'ailleurs avait décrit en 1914 des spores latérales à protoplasme condensé, sessiles, groupées en thyrses et en grappes parfois touffues, qu'il distinguait des arthrospores issues de la désarticulation du thalle. Ces aleuries ont été revues en 1933 par Catanei, dans des cultures sur grains d'orge, où il décrivit de plus quelques fuseaux.

En fait, les aspects microscopiques des cultures diffèrent vraisemblablement avec les souches et peut-être aussi avec le moment de l'année (Joyeux, 1914), ce qui explique sans doute les descriptions légèrement divergentes des auteurs.

\section{$4^{\circ}$ Genre Gtenomyces Eidam, 1880.}

Nous avons isolé six souches présentant les caractéristiques microscopiques des Ctenomyces. En accord avec les synonymies proposées par Emmons (1934), nous les avons étiquetées C. mentagrophytes. Nous avons essayé de dépasser cette diagnose : trois souches appartenaient à la variété mentagrophytes proprement dite, une quatrième à la variété granulosum; quant aux dernières souches, elles ne se rattachaient à aucun champignon du genre antérieurement décrit et leur morphologie macroscopique insuffisamment caractérisée n'incitait vraiment pas à créer à leur occasion de nouvelles variétés. 
Ces six souches provenaient des villes côtières (Bizerte, Tunis, Madhia et Sousse). Elles représentent vraisemblablement aussi des Dermatophytes de provenance étrangère à l'Afrique du Nord.

$5^{\circ}$ Genre Microsporum Gruby, 1843.

Microsporum canis fut identifié trois fois seulement à partir de prélèvements effectués sur des enfants habitant Tunis (l'un Musulman, le second Français, le troisième Italien). L'origine européenne de ces souches est évidente.

\section{Etude comparée des Dermatophytes \\ isolés des teignes du cuir chevelu dans les différents pays de l'Afrique du Nord}

Ces Dermatophytes ont été rassemblés par pays dans le tableau VI (a) avec les dénominations spécifiques proposées par les auteurs.

Dans le tableau VI (b), nous avons groupé ces mêmes champignons en tenant compte des synonymies probables qui intéressent les jordanons d'Achorion schönleini, Trichophyton violaceum et T. glabrum, ainsi que l'ensemble des Trichophyton du groupe cratériforme. Il nous semble évident en particulier que la création de $T$. fuscum et de $T$. nivale, par Lapidus (1950), n'est guère réellement justifiée.

Deux espèces sont par excellence nord-africaines : Trichophyton violaceum et Achorion schönleini. Trichophyton tonsurans est cependant relativement fréquent en Algérie, où il représente $7,39 \%$ des Trichophyton, et plus encore en Tripolitaine $(45,76 \%)$; mais des ensemencements plus nombreux ( 86 prélèvements seulement furent étudiés sur 346) auraient peut-être modifié la fréquence relative des Trichophyton violaceum et des Trichophyton tonsurans dans ce dernier pays.

Dans l'ensemble des pays nord-africains, la rareté des Ctenomyces et des Microsporum est notable. M. audouini, exceptionnel, n'a été isolé qu'en Algérie, d'où proviennent encore, à notre connaissance du moins, toutes les souches de Langeronia soudanensis, à l'exception de celle isolée en Tunisie.

\section{Conclusion}

Nous avons effectué en 1950 et 1953 une enquête sur la teigne en Tunisie : elle a intéressé plus de 24.000 enfants, dont 23.746 formaient un groupe homogène d'écoliers musulmans des kouttabs, des écoles coraniques et franco-musulmanes.

Anv. de Parasitologie, T. XXXI, $\mathrm{x}^{\circ} 4 .-1956$.

$3 e$. 


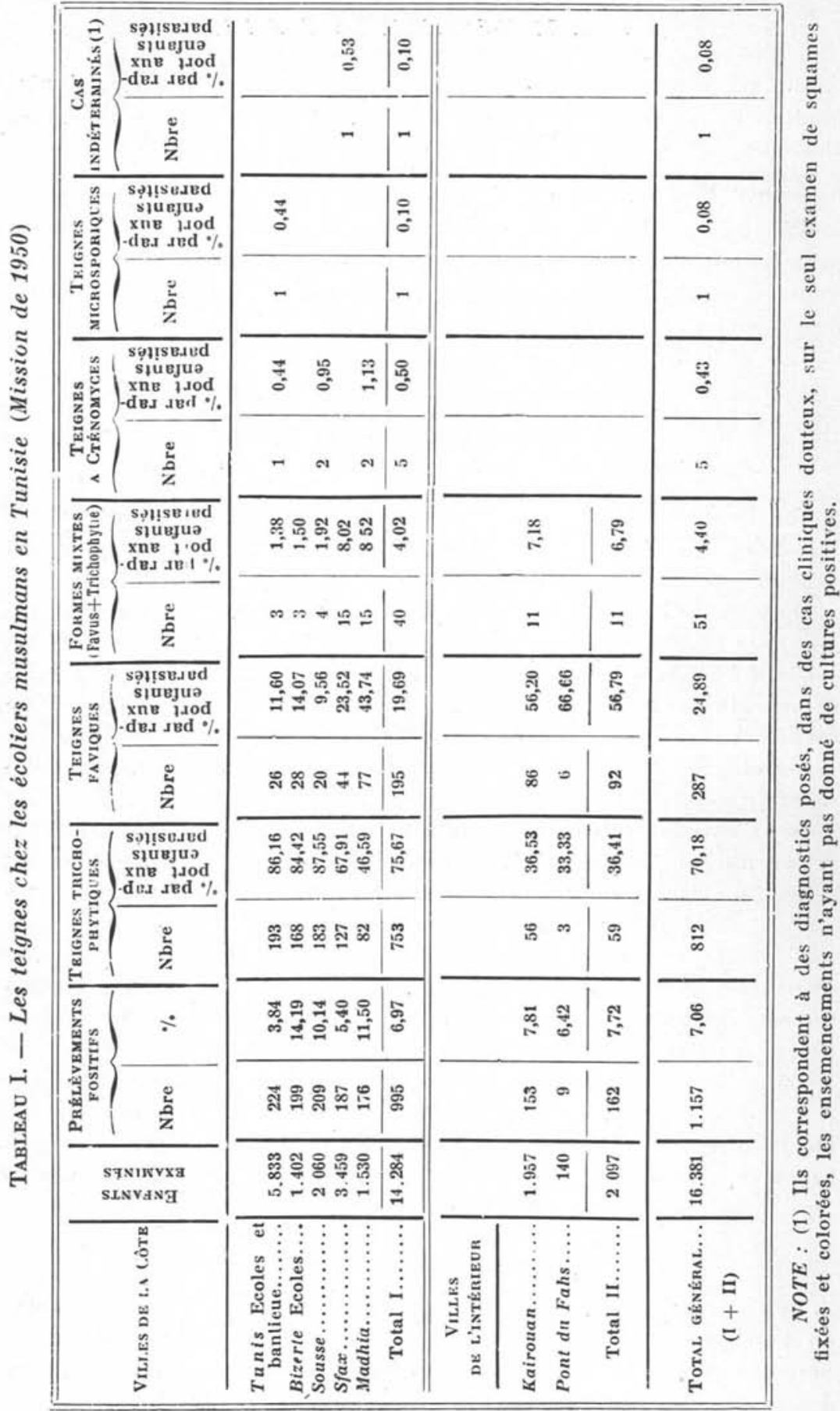




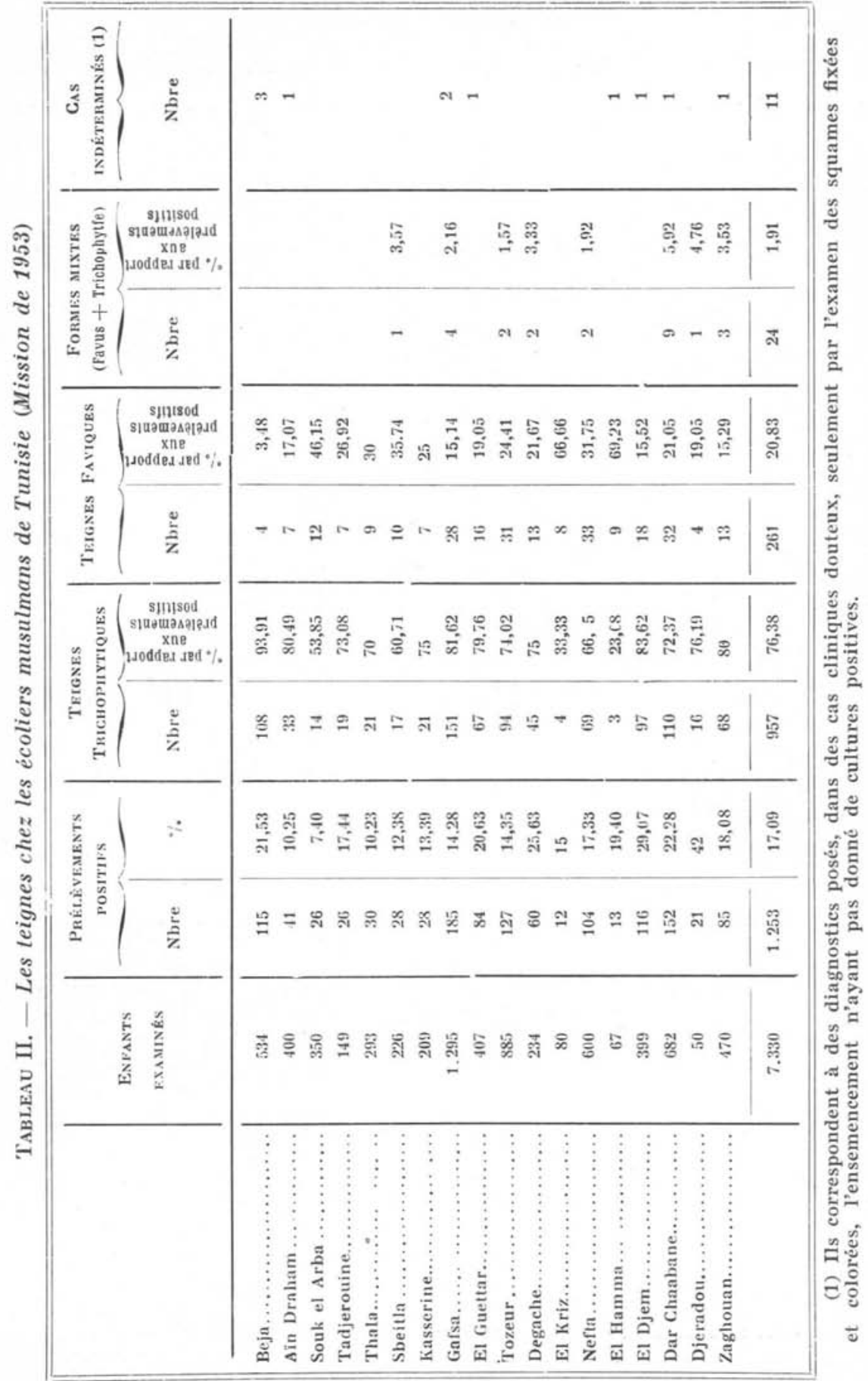

Ann. de Parasitologie, T. XXXI, N ${ }^{\circ} 4 .-1956$. 
Tableau III. - La teigne des écoliers israélites et européens en Tunisie

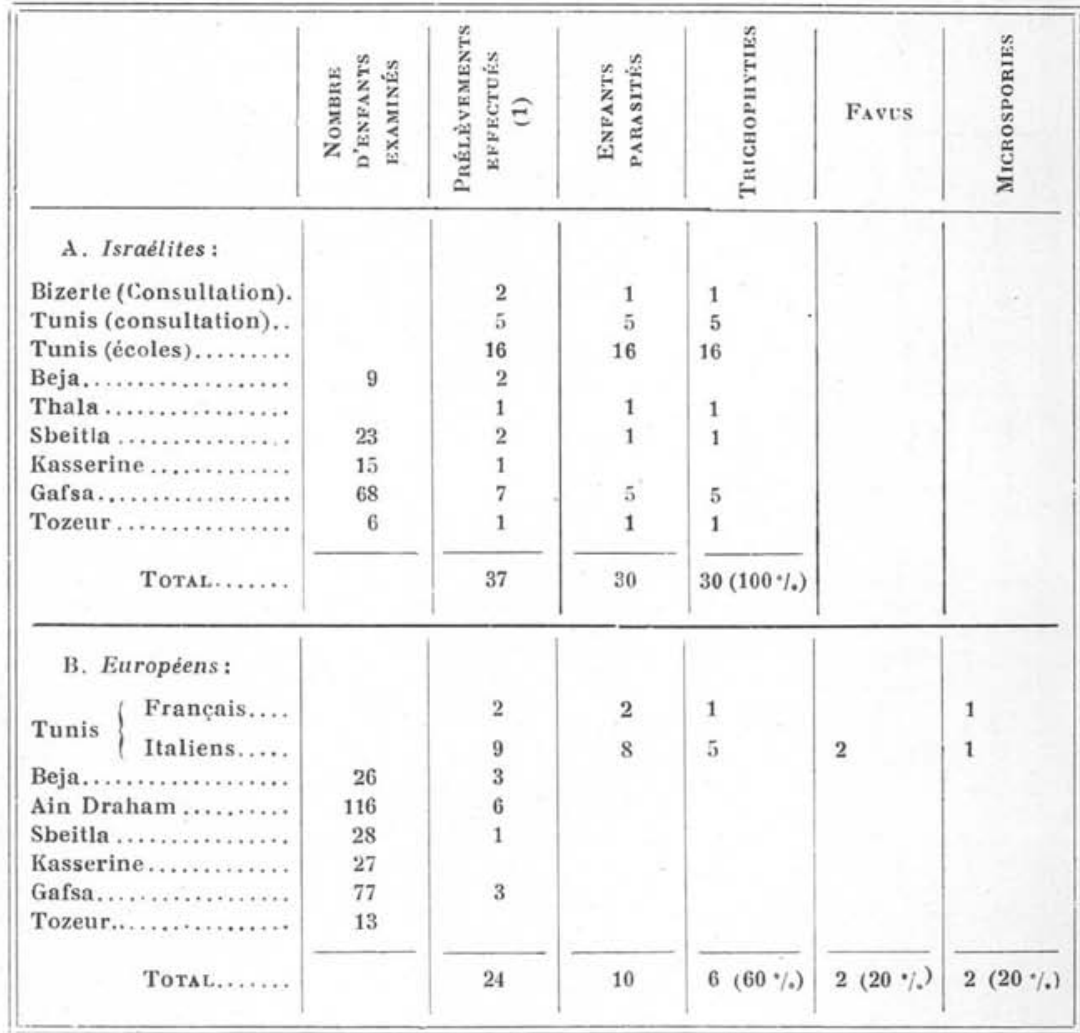

Tableau V. - Les dermatophytes isolés des teignes de Tunisie

\begin{tabular}{|c|c|c|c|c|c|c|c|}
\hline $\begin{array}{l}\text { Púḱlèvemients } \\
\text { Positifs }\end{array}$ & 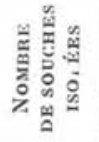 & 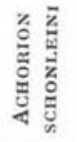 & 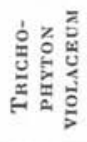 & 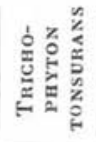 & 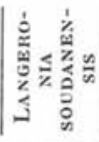 & 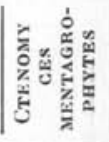 & 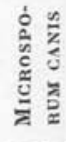 \\
\hline $\begin{array}{c}\text { Musulmans seuls: } \\
2.443\end{array}$ & 1.606 & 544 & 1.037 & 17 & 1 & 6 & 1 \\
\hline $\begin{array}{l}\text { Non musulmans: } \\
40\end{array}$ & 17 & 2 & 13 & 0 & 0 & 0 & 2 \\
\hline Total des souches....... & 1.623 & 546 & 1.050 & 17 & 1 & 6 & 3 \\
\hline
\end{tabular}

(1) Ils ne furent effectués naturellement que dans les cas suspects. 


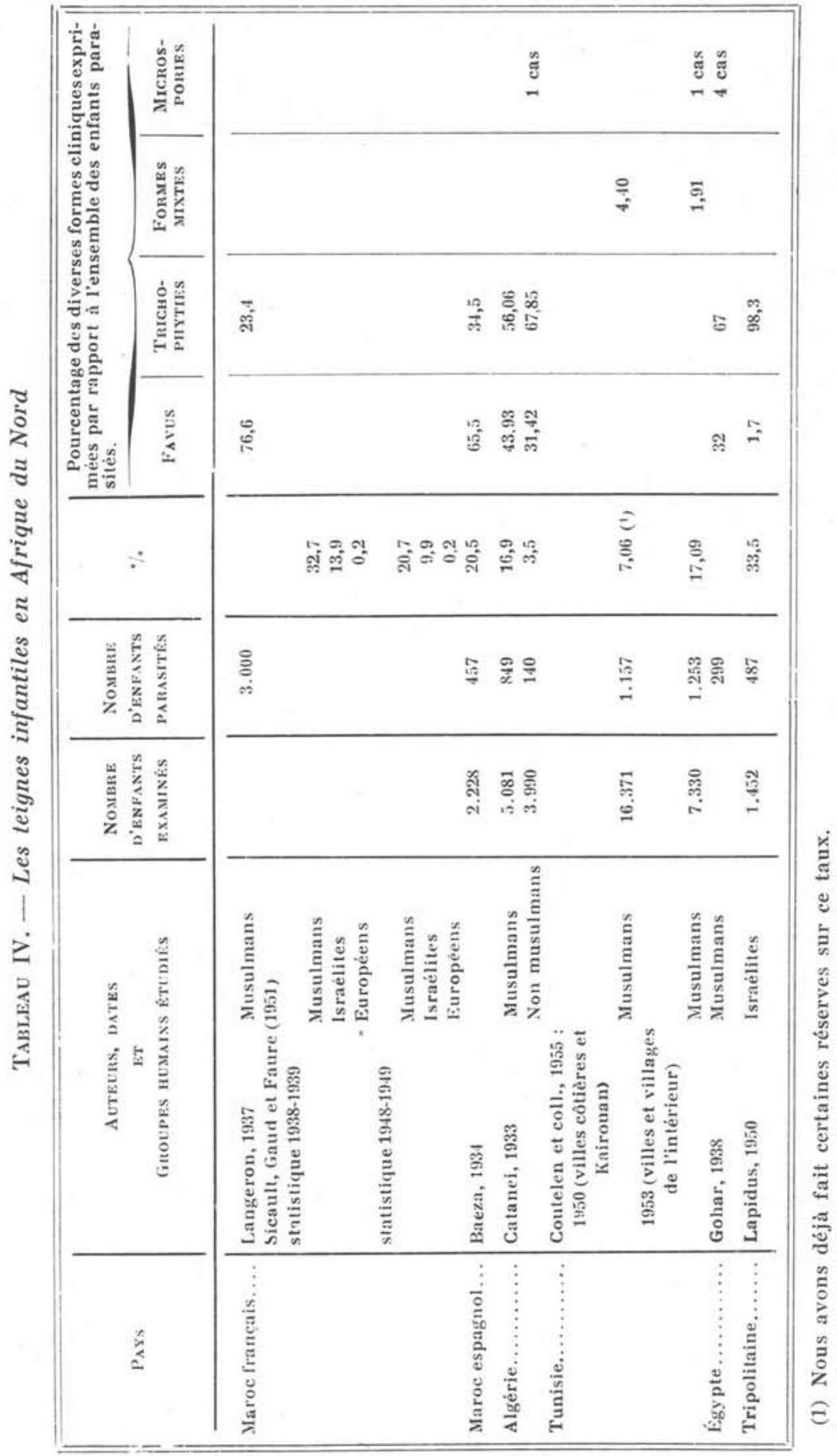




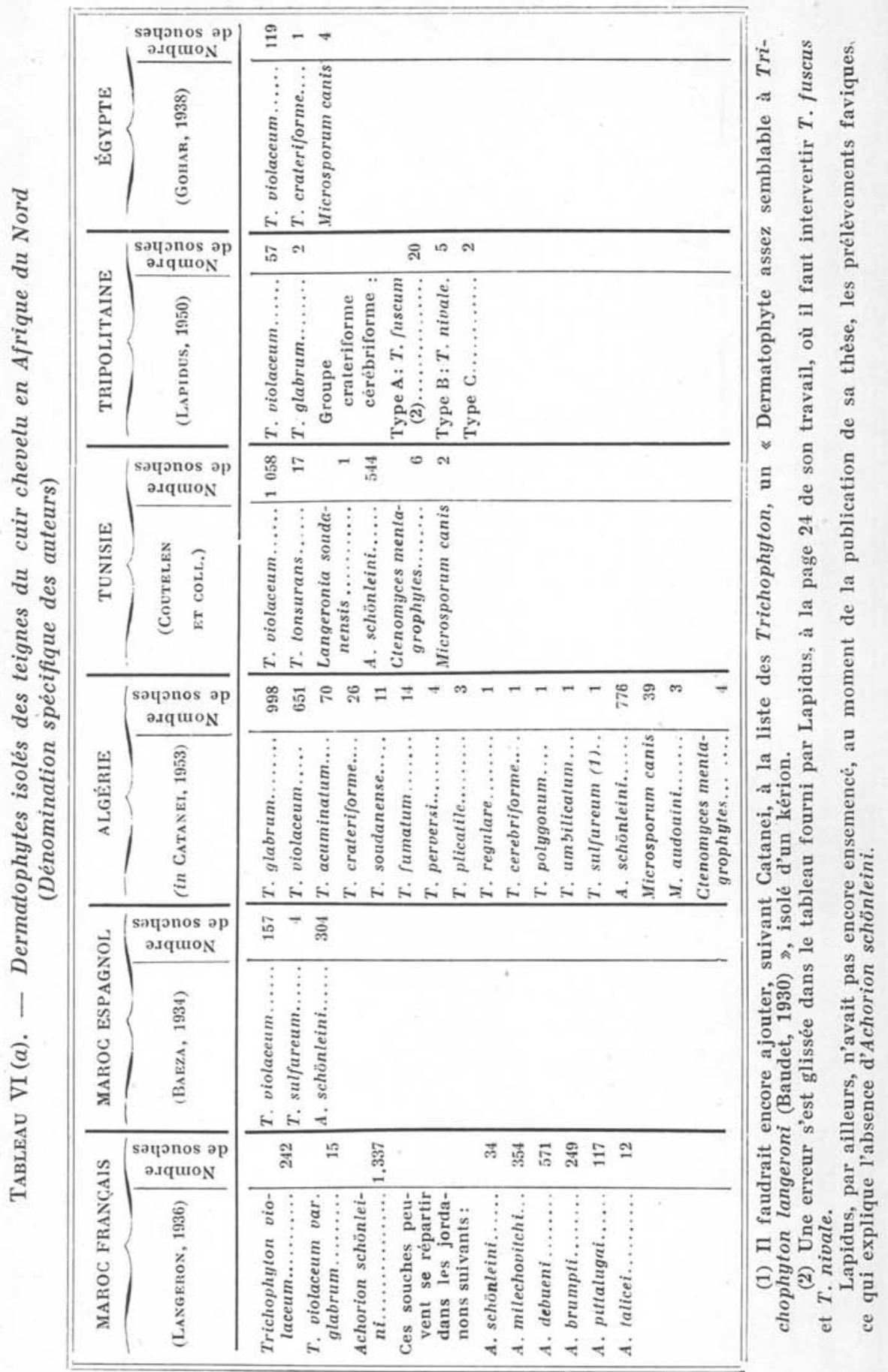




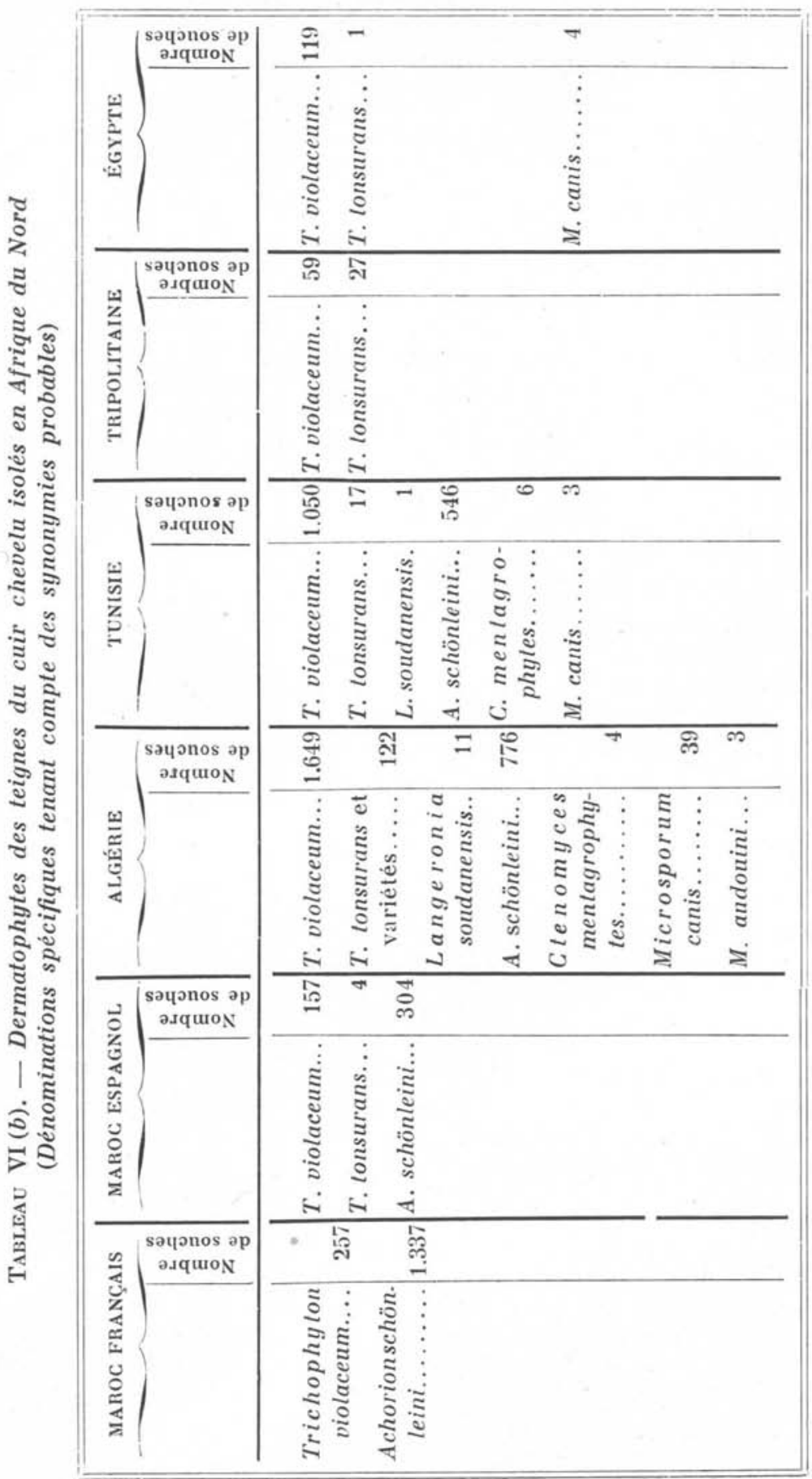


Nous avons constaté :

$1^{\circ}$ Des taux d'infestation globale très variables suivant les localités prospectées et leur organisation sanitaire, allant de $3,84 \%$ à Tunis jusqu'à $42 \%$ à Djéradou ; dans l'ensemble, les villes côtières sont moins parasitées que les villes et villages de l'intérieur. Les filles sont à peu près aussi fréquemment parasitées que les garçons, respectivement $13,99 \%$ et $17,73 \%$; la fréquence globale de la teigne diminue considérablement à partir de 15 ans, ce qui s'explique par la disparition presque totale de la Trichophytie à cet âge.

$2^{\circ}$ Les teignes ne sont représentées que par des Favus et des Trichophyties, parfois associés; les microspories et les teignes suppurées sont exceptionnelles.

$3^{\circ}$ Nous avons observé au total trois fois plus de tondantes trichophytiques que de lésions faviques $(73,22 \%$ des cas contre $22,71 \%$ ). Cependant, le rapport de fréquence des deux formes diffère beaucoup suivant le lieu ; il s'inverse parfois, à Kairouan par exemple.

A la monotonie clinique des teignes tunisiennes correspond la monotonie de la flore mycologique qui les détermine :

Sur 1.623 souches, Trichophyton violaceum fut isolé 1.050 fois et Achorion schönleini 546 fois. Les autres espèceś sont représentées par $T$. tonsurans (17 souches généralement atypiques), Langeronia soudanensis (1 souche), Ctenomyces mentagrophytes (6 souches dont 2 atypiques) et Microsporum canis (3 souches).

Au total, les teignes infantiles de Tunisie se réduisent dans la pratique au Favus déterminé par A. schönleini et à la Trichophytie déterminée par $T$. violaceum.

L'endémie teigneuse de la Régence est donc à l'image de celle de l'Afrique du Nord tout entière, où seule la Tripolitaine semblerait se singulariser par la rareté du Favus et la fréquence des Trichophyties ayant, dans un tiers des cas, des Trichophyton cratériformes comme agents.

\section{Remerciements}

A la fin de ce travail, nous sommes heureux de remercier très vivement M. le Professeur Jean Roche, Directeur de l'Institut des Hautes Etudes Tunisiennes, son secrétaire général M. Pignon, ainsi que M. le Docteur A. Ottavy, Inspecteur général de l'Hygiène scolaire en Tunisie, dont l'accueil très cordial, l'aide matérielle et les conseils nous furent indispensables pour organiser nos tournées. 
Et nous ne voudrions pas terminer sans assurer aussi de notre gratitude et de notre meilleur souvenir, tous les médecins de la Santé publique, directeurs de dispensaires polyvalents, toutes les infirmières et assistantes sociales, qui ne ménagèrent jamais leur temps, ni leur peine, afin de nous venir en aide.

Notre souvenir reconnaissant va aussi à Mlle G. Cordier et au Docteur P. Durand pour l'accueil si amical que nous avons toujours trouvé à l'Institut Arloing et à l'Institut Pasteur de Tunis.

\section{BIBLIOGRAPHIE}

BaEz (M.). - 1934. Note statistique sur les teignes au Maroc Espagnol. Ann. Parasit., XII, p. 405.

Catanei (A.). - 1933. Etudes sur les teignes. Arch. Inst. Pasteur d'Algérie, XII, p. 267.

1950. Les teignes en Afrique du Nord. Répartition, formes cliniques et évolution, champignons parasites, traitement. Maroc Médical, p. 955.

1953. Sur la valeur de classification des caractères culturaux du champignon du favus. Arch. Inst. Pasteur d'Algérie, XXXI, p. 54.

Conant (N. F.), Smith (O. T.), Baker (R. D.), Callaway (J. L.) et Martin (O.). 1954. Manual of clinical Mycology, Philadelphie et Londres, W. B. Saunders Company, $2^{\circ}$ édition.

Emmoxs (C. W.). - 1934. Dermatophytes natural grouping based on the form of the spores and accessory organes. Arch. Dermat. Syph., XXX, p. 337.

GoHar. - 1938. The first survey of ringworm in Egypt. J. Trop. Med. and Hyg., XLI, p. 229.

Josserand (M.). - 1951. Remarques moroses sur la notion d'espèce en mycologie. Bull. trim. de la Soc. Mycologie de France, LXVII, p. 1951.

Joyeux (C.). - 1914. Contribution à l'étude des teignes africaines : Trichophyton soudanense. Arch. Parasit., XVI, p. 447.

Langeron (M.). - 1937. Observations statistiques et mycologiques sur les teignes humaines au Maroc. C.R. Acad. Sc., CCIV, p. 372.

1945. Précis de Mycologie, Masson et $\mathrm{C}^{\text {ie }}$ éditeurs, Paris.
- et Mrochevrtch (S.). - 1930. Morphologie des Dermatophytes sur milieux naturels et milieux à base de polysaccharides. Essai de classification (deuxième mémoire). Annales de Parasit. Hum. et Comp., VIII, p. 465.

- et Vanbreusechem (R.). - 1952. Précis de Mycologie, Masson et $\mathrm{C}^{\text {ie }}$ éditeurs, Paris,

Lapidus (R.). - 1950. Les teignes en Tripolitaine. Thèse Doct. Méd. Paris.

Sabouradd (R.). - 1910. Les teignes, Masson et $\mathrm{C}^{\text {le }}$ éditeurs, Paris.

Sicault (G.), Gaud (J.) et Faure (J.). - 1951. Les teignes au Maroc. Bull. de l'Institut d'Hygiène au Maroc, XI, p. 165.

Vanbreuseghem (R.) et VAN Brussel (M.). - 1950. La terre, facteur de mutation d'un Dermatophyte, Langeronia soudanensis (Joyeux, 1912), Vanbreuseghem, 1950. Annales de la Soc. belge de Méd. Trop., XXXII, p. 79.

(Laboratoire de Parasitologie, Faculté de Médecine de Lille). 